\title{
Using Participatory Activities with Seniors to Critique, Build, and Evaluate Mobile Phones
}

\author{
Michael Massimi \\ Department of Computer Science \\ University of Toronto \\ Toronto, Ontario, Canada \\ mikem@dgp.toronto.edu
}

\author{
Ronald M. Baecker \\ Department of Computer Science \\ Knowledge Media Design Institute \\ University of Toronto \\ Toronto, Ontario, Canada \\ rmb@kmdi.toronto.edu
}

\author{
Michael Wu \\ Department of Computer Science \\ University of Toronto \\ Toronto, Ontario, Canada \\ mchi@dgp.toronto.edu
}

\begin{abstract}
Mobile phones can provide a number of benefits to older people. However, most mobile phone designs and form factors are targeted at younger people and middle-aged adults. To inform the design of mobile phones for seniors, we ran several participatory activities where seniors critiqued current mobile phones, chose important applications, and built their own imagined mobile phone system. We prototyped this system on a real mobile phone and evaluated the seniors' performance through user tests and a real-world deployment. We found that our participants wanted more than simple phone functions, and instead wanted a variety of application areas. While they were able to learn to use the software with little difficulty, hardware design made completing some tasks frustrating or difficult. Based on our experience with our participants, we offer considerations for the community about how to design mobile devices for seniors and how to engage them in participatory activities.
\end{abstract}

\section{Categories and Subject Descriptors}

H5.2 [Information Interfaces and Presentation] User Interfaces Evaluation/Methodology, Prototyping, User-centered design,; D.2.1 [Software engineering] Requirements/Specifications - Elicitation methods.

General Terms: Human Factors, Design.

\section{Keywords}

Seniors, elderly, mobile phones, mobile devices, accessibility, participatory design, scenarios, usability, guidelines.

\section{INTRODUCTION}

Mobile phones can be great tools for older people, but are usually designed with younger people in mind. Glamorous, thin, and computationally powerful mobile phones dominate a marketplace where the trend is towards smaller, trendier, and faster. In this paper we dispel the misconception that mobile phones are for younger

Permission to make digital or hard copies of all or part of this work for personal or classroom use is granted without fee provided that copies are not made or distributed for profit or commercial advantage and that copies bear this notice and the full citation on the first page. To copy otherwise, or republish, to post on servers or to redistribute to lists, requires prior specific permission and/or a fee.

ASSETS'07, October 15-17, 2007, Tempe, Arizona, USA.

Copyright 2007 ACM 978-1-59593-573-1/07/0010...\$5.00. people, and instead suggest steps we can take towards designing mobile phone software for and with senior citizens.

Sheer numbers indicate the importance of designing better mobile phones for seniors. The elderly are a large and growing percentage of the worldwide population. The United Nations projected that by 2050 , people over 60 will account for $21 \%$ of the world population [24]. This growth will be most pronounced in developing countries where mobile phones are rapidly penetrating the population [6]. From the technology side, there are now over 2 billion mobile phone users worldwide, with some developed countries reaching penetration rates over $100 \%$ due to multiple phones per person [17]. Continuing to focus mobile device design on younger people will exclude a very large demographic from successful product adoption and use.

Two trends in creating "senior-friendly" phones focus on decreased sensorimotor skills and a reduction in complexity via a decrease in functionality. For example, the Silverphone Easy5 mobile phone [20] has only a power button, a talk button, and 5 pre-programmed phone numbers bound to individual buttons (Figure 1, right). For seniors with dementia this may be appropriate, but for normally aging seniors it is too restrictive. At the same time, families are giving their old mobile phones (such as the Nokia 6185 in Figure 1, left) to their elderly family members [21]. These repurposed phones often have large keys and monotone, but crisp, displays.

While mobile phones should appeal to seniors, we also discourage hasty, single-function, or repurposed solutions. In order to create accessible and useful mobile phones, we must first develop a better
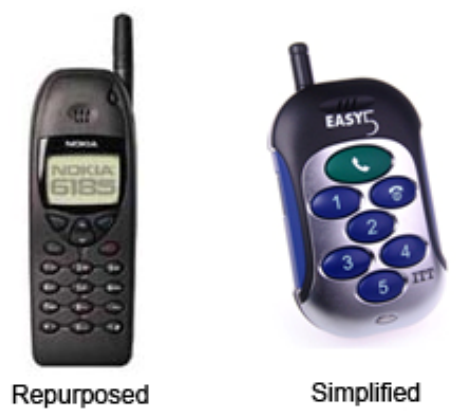

Figure 1. The Nokia 6185 (left), released in 1999, illustrates the trend to repurpose phones for seniors [16]. The Silverphone

Easy5 mobile phone (right) only has 7 keys and exemplifies the trend towards oversimplification [20]. 
understanding of seniors' needs and capabilities. In this paper, we contribute to this understanding in the following ways:

- We describe the functions and hardware characteristics that the seniors would include in their ideal mobile phone (Section 3).

- We co-designed a mobile phone system with a group of seniors, which we then prototyped and tested. The system is called Recall and includes a large subset of applications the seniors considered important. We describe the results of a set of usability tests with the design team. We show that while considerable barriers existed, motivated seniors were not overwhelmed by the phone software. Seniors surprised themselves with their ability to use the phone (Section 4).

- We highlight methodological considerations when using participatory activities with seniors (Section 5).

- We offer design considerations for mobile devices for seniors. These considerations come from our usability tests and 6 months of interaction with the team of seniors (Section 6).

While this study examined a mobile PDA phone, many aspects of mobile phone design can be applied to a wider range of mobile devices because of similar form factors, carrying considerations, and hardware components (e.g., screens). The class of devices which can benefit from insight into mobile phone design includes personal digital assistants (PDAs), handheld entertainment systems, tablet PCs, and ultra-mobile PCs.

We also feel mobile device interaction is an important topic for the ASSETS community. Important previous work dealing with mobile devices for special needs populations has taken an approach where the mobile device serves as a platform upon which to deliver a novel application. Examples include wayfinding applications [12, 22], mobile memory aids [19], and keyboard/mouse alternatives [15]. One exception to the application-centric viewpoint is Leonard et al.'s work studying age-related macular degeneration and its implications for legibility on mobile devices [10]. While mobilerelated efforts to create new applications are important, there is still a great deal to be done to make existing systems more accessible. This includes developing new user interfaces, re-thinking hardware design, and reinventing common applications.

\section{METHOD}

Researchers have uncovered important considerations about seniors' attitudes towards mobile phones through several techniques. Keating et al. collected ethnographic data through direct observation, individual interviews, group interviews, and online discussion forums [7]. More structured focus groups have yielded

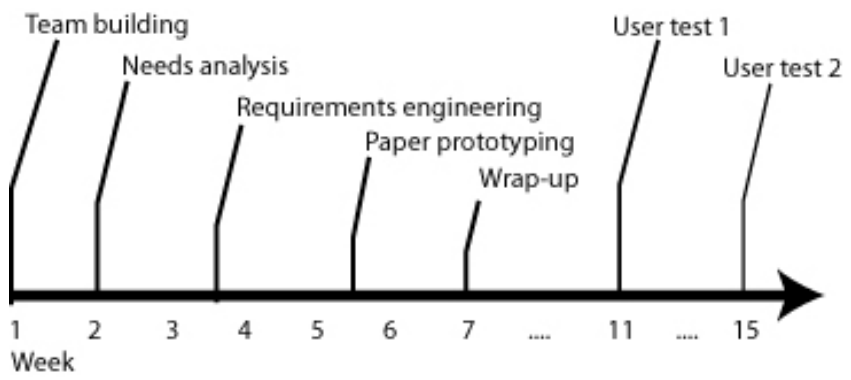

Figure 2. Timeline of participatory activities and tests.

\begin{tabular}{|l|l|l|l|l|l|}
\hline & Age & Sex & $\begin{array}{l}\text { Mobile } \\
\text { phone }\end{array}$ & $\begin{array}{l}\text { Comfort } \\
\text { with PCs }\end{array}$ & Difficulties \\
\hline P1 & 76 & F & Yes & Novice & Eyesight \\
\hline P2 & 71 & F & Yes & Novice & Hearing \\
\hline P3 & 78 & F & No & Moderate & Tremors \\
\hline P4 & 55 & F & No & Moderate & Eyesight \\
\hline P5 & 86 & F & No & Expert & $\begin{array}{l}\text { Hearing, } \\
\text { mobility }\end{array}$ \\
\hline
\end{tabular}

Table 1. Participants in the participatory activities and usability tests.

interesting results $[5,9]$ regarding preferences from a team of older women in the UK, where attitudes towards mobile phones arguably differ from North American populations. However, these methods do not give the seniors the opportunity to actually change the way the mobile phone in question operates.

In our study, we use guided participatory activities to elicit feedback from seniors about mobile phones. These activities allow the seniors' feedback to feed directly into the design of new mobile phones. Participatory methods have been used to understand seniors' needs with respect to several other technologies, including websites [3], minimalist tutorials and simulation environments [18], usability guidelines [8], and game concepts [1]. Participatory methods have established themselves as promising for working with other populations, including people with amnesia [25] and aphasia [13]. These methods, originated in Scandinavia, enjoy a rich history stemming from cooperative and inclusive design [4].

\subsection{Participants}

We recruited 5 participants (P1 to $\mathrm{P} 5$ ) by distributing flyers to community centers and hospitals with senior outreach programs ${ }^{1}$. Interestingly, only women volunteered (similar to Kurniawan's focus group [9]). We did not screen participants for inclusion in the study. Table 1 contains ages and reported sensorimotor difficulties. Participants sometimes missed the weekly design meetings due to scheduling conflicts. P2 and P5 both used hearing aids. P3 suffers from familial tremors and cannot exert fine-grain motor control and uses pencil grip-enhancers to write. P5 walks with a cane and has limited mobility. All expressed interest in learning about mobile phones and technology.

\subsection{Group Activities}

Over the course of 7 weekly group meetings, we conducted 3 major software design activities: needs analysis, requirements engineering, and paper prototyping. We interviewed participants in both group and individual settings throughout. We also allotted time to build trust [3], teach seniors about current mobile phone technology, and socialize. We present here the way in which we conducted these activities; the results of the activities are reported in Section 3.

\subsubsection{Needs Analysis}

The needs analysis consisted of two primary activities: mobile phone critiques, and scenario-based design [2].

\footnotetext{
${ }^{1}$ This work was approved by the University of Toronto Research Ethics Board, protocol number 15937.
} 


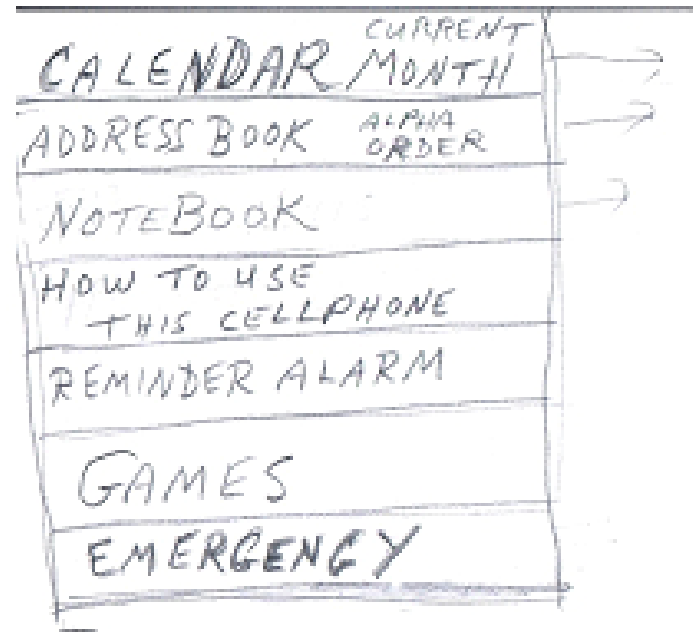

Figure 3. The "Main Menu" as sketched out by the design team contains the most important application areas.

To conduct the mobile phone critiques, we presented a slideshow containing photographs of popular mobile phones. We purposefully chose PDA phones for this activity because of their variety of form factors (e.g., clamshell, candy-bar, slide-out) and styles of interaction (e.g., stylus, jog-wheel, thumb-board). We highlighted specific features of each phone and encouraged seniors to make critical comments about their designs [23]. We also prompted discussion by asking the group questions about form factor, interaction styles, and aesthetics. In the end, our design team wrote a list of desirable and undesirable features for each phone on a whiteboard. We then collaboratively ranked these features in order of importance.

Our scenario-based design activity consisted of dividing the group into two teams. By pairing P3 (who has tremors) with another senior on the team, she was able to participate in this writing and drawing focused activity. Each team created a series of illustrated scenarios describing situations where having a phone would be useful as a personal organizer or memory aid. We chose this emphasis to encourage the seniors to think of the mobile device as more than simply a telephone. After creating 3 scenarios, the teams presented their scenarios and illustrations to the entire group, prompting group discussion.

\subsubsection{Requirements Engineering}

Rather than simply critiquing design and understanding phone use cases, we had the seniors develop their own mobile phone software. We anticipated that by doing so, the seniors would highlight commonly overlooked problems that do not readily spring to mind during interviews or focus groups.

The team brainstormed a list of function areas that they would like in their ideal mobile phone. We wrote these ideas on the whiteboard and organized them into categories. We then asked the team to describe what would happen when they powered up the phone. What options would there be available? The team created a list of "main menu" options that they felt were important to include in a phone for a senior citizen (Figure 2). Seniors differed in their opinions about what was important to include on this menu (see Discussion).

\subsubsection{Paper Prototyping}

The team created paper prototypes of two applications denoted in the main menu: the calendar, and the address book. The team chose to create paper prototypes of these applications because they were perceived to be more important than the rest.

To conduct paper prototyping, we used the PICTIVE method [14]. We provided paper UI controls which could be placed on cut-outs of mobile phones. We also provided sticky notes, markers, pencils, pens, glue, and tape for the participants to construct their interfaces. We encouraged them to use whatever means they felt was most appropriate for building the UI of their mobile phone.

\subsection{Measurements}

We collected data from questionnaires and interviews with the participants throughout the entire design process. Questionnaires consisting of 7-point Likert scale items assessed their perceptions of the activity experience, and interviews supplemented these questionnaires with personal narratives.

\section{RESULTS OF GROUP ACTIVITIES}

\subsection{Function Areas}

From the group activities, the seniors identified 7 main areas of functionality they would like in a mobile phone (see Figure 3). We report these here to illustrate the breadth of interest the seniors exhibited. Contrary to common misconceptions, seniors desired a variety of applications beyond simply placing phone calls. Further, these areas can help focus the scope of application development for designers who are interested in building phones for seniors.

1. Calendar: Stores appointments, birthdays, and long-term information and reminders.

2. Address book: Contains contact information for friends, family, and organizations (e.g., doctor's offices, auto associations).

3. Notebook: Stores short-term notes such as shopping lists.

4. How to use this cell phone: Contains clear, to-the-point, numbered checklists of how to operate the phone "written by a native English speaker" (P1).

5. Reminder alarm: Quickly sets short-term reminders for activities like remembering to take out the laundry or return a phone call.

6. Games: Contains parlor games like Solitaire, Poker, and other simple games similar to those found on desktop computers. The team also enjoyed playing Sudoku and solving crossword puzzles. They thought such games would be good "distractions"; they felt games could help reduce stress induced by, for example, waiting at the doctor's office. The team also recommended including games to keep mentally sharp or "exercise your brain." 
7. Emergency: Stores information for first responders, lists current medications, and automatically calls 911 and a pre-set list of family members.

Opinions about which applications to include on the phone varied. P5 saw the phone as an emergency device, with all else secondary. Her attitude towards games was dismissive. P2 and P4, on the other hand, felt that games would be a great addition and saw the phone as a platform for personal information management and communication.

\subsection{Hardware Features}

The team also listed a number of considerations they take into account when choosing mobile phones. We collected these on a whiteboard and subsequently discussed each one. This list illustrates some important considerations for choosing a mobile phone for seniors and also corroborates findings from Kurniawan's focus group [9].

1. Large buttons: The seniors felt that large buttons were extremely important to any device they would use.

2. Screen/text size: The seniors noted they wanted to zoom in on small text and needed a large, clear, bright screen.

3. Grip: Seniors worried about being unable to grasp the phone properly and securely.

4. Selection mechanism: Seniors disliked directional pad selection mechanisms often found in the center of mobile phones. They preferred a jog wheels (as on a Blackberry).

5. Weight: Seniors preferred rugged phones that could withstand drops and scratches, but did not want them to be too heavy.

6. Hearing aid compatibility: Phones should be compatible with hearing aids and have a wide range of volume levels.

\subsection{Prototype}

Based on the features describe above, we built a prototype system that the team named Recall. We describe the hardware and software that comprise the Recall system here.

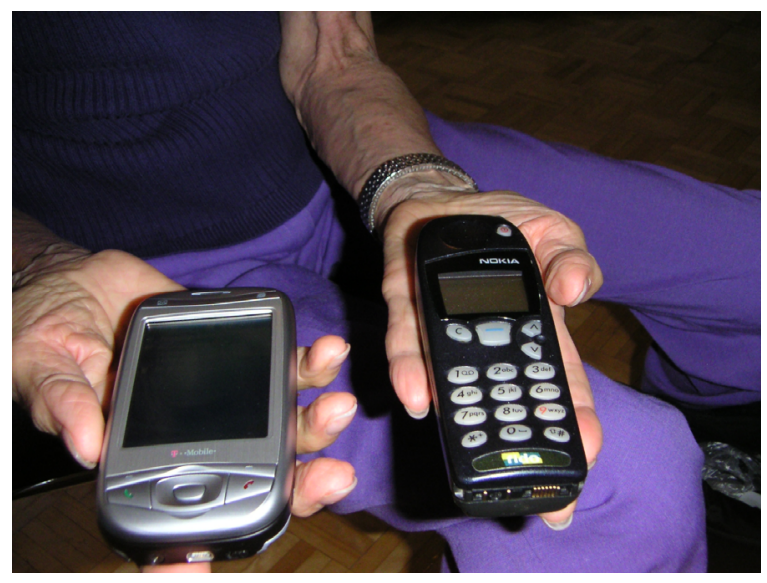

Figure 4. P1 compares the i-Mate K-JAM used in the study (left) with her own "hand-me-down" Nokia mobile phone (right).

For the hardware platform, we selected the iMate K-JAM mobile phone (Figure 4) because it met most of the hardware features describe above. The K-JAM features a slide-out QWERTY keyboard, a 240x320 pixel resolution, and a touch-sensitive screen allowing interaction with a stylus or finger. It runs Windows Mobile 5 OS.

The seniors envisioned a system with 7 main functions. Due to time constrains we could only prototype 5 of these. Of these 5 , the seniors created paper prototypes for 2 and we created 3 based on discussion. The calendar paper prototype was not sufficiently different from the built-in Windows Mobile Calendar application to warrant rebuilding it.

Figure 5 demonstrates the evolution of the Address Book software. We moved from a paper prototype to a software version. In comparison we show the business-oriented Contact manager. All prototype software was written in C\#.NET using the Pocket Outlook Object Manager API, allowing interoperability with other preexisting phone functions. We edited the registry to change the Start Menu items to reflect the Main Menu that the team sketched.
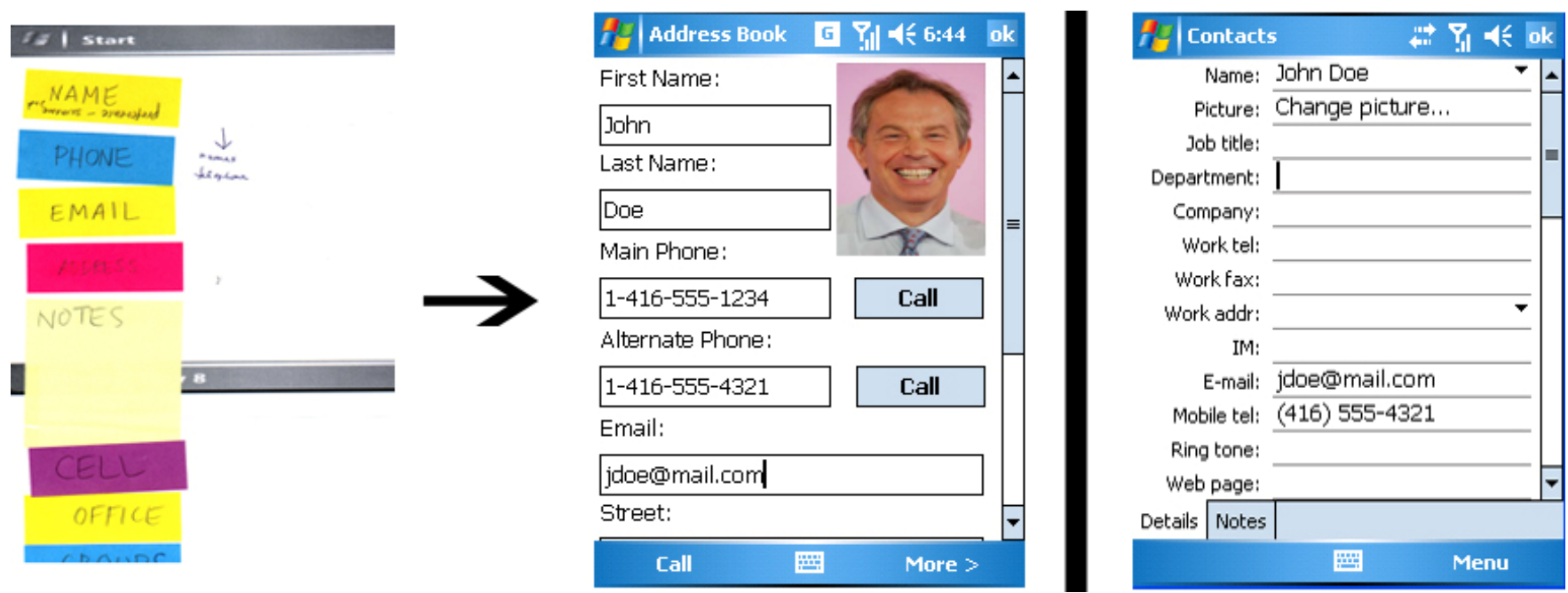

Figure 5. The paper prototype for the address book (left) was developed into the Address Book prototype software (center). It is simpler than the business-oriented Contact Manager in Windows Mobile 5 (right). 


\begin{tabular}{|l|l|l|l|l|l|l|}
\hline Item & P1 & P2 & P3 & P4 & M & SD \\
\hline Recognize custom? & Y & N & N & Y & & \\
\hline Liked custom & 4 & 4 & 6 & 6 & 5.00 & 1.15 \\
\hline Liked built-in & 3 & 5 & 3 & 5 & 4.00 & 1.15 \\
\hline Use custom again & 6 & 4 & 6 & 6 & 5.50 & 1.00 \\
\hline Use built-in again & 2 & 5 & 6 & 5 & 4.50 & 1.73 \\
\hline
\end{tabular}

met with them 2-3 times per week to note where they were having trouble and to answer their questions. This long-term deployment, while small, shed light on barriers to adoption.

\section{PARTICIPATORY ACTIVITY CONSIDERATIONS}

Based on our sessions with the seniors, we suggest considerations for researchers using participatory methods with this population. Problems often stemmed from sensorimotor problems, as demonstrated below.

Table 2. Results from the usability tests show a slight preference for the system the seniors created. Scores range from 1 (strongly disagree) to 7 (strongly agree).

\section{USER TESTS AND DEPLOYMENT}

Following the 7 group meetings, we met with 4 participants individually at a location of their choosing for 2 interviews at roughly 30 and 60 days following the end of the group activities. We suggested they meet us at a location where they would use a mobile phone often. We interviewed seniors in their homes, at neighborhood cafes, and at volunteer-run senior centers. This allowed us to ask questions about imagined use each of those places (e.g., "What applications do you think you would use most here?"). We met the participants at the same place for both interviews. P5 could not participate in the remainder of the study for personal reasons.

In the first interview, the senior explored the phone and asked questions about its operation. We observed the senior while she used the phone, and took notes about problem areas. Specific functions that the seniors explored included placing and receiving phone calls, creating notes, adding items to the calendar, adding contacts, and taking photographs. They were also fond of the onetouch voice recorder feature. We did not ask seniors to perform specific tasks at this time, but rather observed problems they had while working with the phone and its built-in applications.

In the second interview, we included the prototype Recall software. We asked each participant to complete a series of tasks with the Address Book application. We chose this application because the seniors designed a paper prototype of it and because Windows Mobile Contact manager could serve as a comparison.

Each senior used both the Windows Mobile Contact manager and the custom Address Book system. We counterbalanced the presentation order across participants.

Participants completed the same task on both address books. The task involved adding a new contact, taking a picture, saving the contact, sorting the list of contacts, editing an existing contact, and deleting a contact. These functions were selected because they were the features that the seniors included in the paper prototype. With the exception of search, the task was a comprehensive tour of the custom Address Book prototype.

Table 2 displays the results of a questionnaire given at the end of the session $(n=4)$. For items $1-4$, Likert responses ranged from 1 (Strongly Disagree) to 7 (Strongly Agree). After the questionnaire, we asked each participant to identify the design that they helped create. Of the 4 participants, 2 could not identify the design that they helped to create 3 months earlier.

Beyond the usability testing, 2 seniors chose to work with the phone for 4 additional weeks and received training on its operation. We

1. Provide alternative activities: When conducting activities that require the seniors to draw, sketch, or move about, ensure that there is are alternative ways to incorporate people whose disabilities might prevent them from participating fully. For instance, while some people create a paper interface, ask participants with arthritis or tremors to instead debate which parts of pre-existing interfaces they like best and why. Some people also simply prefer to work alone or in a pair as opposed to a larger group.

2. Create temporary subgroups to overcome deficits. When working with seniors with multiple minor deficits and varying comfort levels with technology, facilitators can use particular groupings to overcome deficits. For example, by pairing P3 (a woman with tremors) with P1 (a woman who enjoyed writing), P3 could participate in a writing-intensive task. Likewise, pairing P5's computer experience with P2's discomfort with computers created a peer teaching situation.

3. Minimize crosstalk: Auditory problems exacerbated difficulties understanding streams of conversation, and also led to people talking at the same time. The facilitator must make sure that one person speaks at a time, and that this person can be heard by all other participants.

4. Make participation an institutional affair. Participatory activities occurring in businesses or among community groups benefit from a pre-existing sense of shared interests and responsibilities. In Ellis and Kurniawan's study, the seniors all lived in the same residential community [3]. In Wu, Baecker, and Richards's study, the amnesic individuals were members of a pre-existing weekly clinical program [25]. Our team members came from the community with no pre-existing relationships. Each senior brought different goals for what they would like to accomplish, and had varying levels of sensorimotor difficulties and computer experience. These variances caused frustration among group members at times. Because participatory studies are still emerging as a common practice, seniors who expected to take part in a laboratory trial seemed to be confused about what to expect from the program. By making the study part of a larger regiment of care, or part of ongoing community service, the seniors have a context in which to understand participatory design.

5. Provide activity structure: We originally allowed seniors to dictate their own course throughout the process, and gave them freedom to change the agenda. The seniors, however, wanted the organizers to provide the structure. In the questionnaire distributed at the end of the group meetings, they disagreed that meetings were too structured and preferred to be given an activity to try rather than to define their own course. 
6. Speed up or down to suit the group: We originally thought we could complete in 5 weeks, but during the process, the seniors seemed to need more time. At the end, however, most participants felt that we proceeded too slowly through the design process. One even wished that meetings were twice a week, as she would often have new ideas during the week and wanted to act on them quickly. Individual and group sessions together may help address this (see next guideline).

7. Blend individual and group sessions: Sessions between researcher(s) and an individual allow participants to confidentially share their thoughts about the group meetings. Participants who want to "speed up" can use this time to elaborate and share, while participants who want to "slow down" can use this time as a review and opportunity for mutual understanding. Finally, individual sessions allow more opportunities for prototype evaluation, and may lead to more productive group meetings.

\section{DESIGN CONSIDERATIONS FOR MOBILE DEVICES FOR SENIORS}

We offer the following suggestions for designing mobile devices for seniors based on the results of our study. These suggestions span both hardware and software aspects of the phone design.

1. Eliminate buttons on the sides and rear of devices. In an attempt to press a button on the front of the phone with their dominant hand, seniors often pressed other buttons on the back or side of the phone with their non-dominant hand. As a result, pressing button A would sometimes cause the calendar to open; sometimes, the senior thought they were pushing A but were also pushing B by accident, causing a configuration menu to open. This violates Lewis's Previous Action heuristic [11].

2. Avoid soft keys. Seniors had difficulty building mental models when buttons resulted in different actions depending on the application. Due to limited device size, manufacturers use "soft keys," to execute particular actions depending on the application. For example, upon booting the device, these may say "Calendar" and "Contacts." Once launching the calendar, however, these change to "New" and "Menu." The changing labels on these buttons prevented seniors from constructing appropriate mental models [11]. Better choices may include on-screen numbered selections (e.g., Press 1 for Calendar) or direction manipulation through a stylus.

3. A single, consistently placed button for returning to the home state should be included. Seniors learned by understanding deviations from the Today screen - the main screen in Windows Mobile that appears upon booting the device. However, the method for returning to this screen varied depending on the application. For example, the red "hang-up" button sometimes ended a call, and sometimes returned the user to the Today screen (i.e., closed the current application). This meant that seniors created inaccurate mental models about how to return to a familiar state.

4. Consider human support networks. Seniors emphasized the importance of proper documentation. However, having a human support network is more important. Participants often wanted to talk to a friend or expert to work through problems rather than attempt to understand complex technical manuals. $\mathrm{P} 4$, whose friends had mobile phones, often relied on them to teach her how to use her phone. P2, on the other hand, had no friends who used mobile phones, and gave up quickly. Mobile phone manufacturers targeting seniors should include especially high-quality tech-support lines.

5. Include several input modalities. Seniors varied in their favored input modality but found entering text difficult overall. Some liked using the hardware keyboard, while others preferred using a stylus to tap on the on-screen keyboard. For P3, her tremors made using the stylus and keyboard both quite difficult. She became so frustrated that her usability test ended early. Likewise, P1 remarked "This is a nightmare! I cannot do this! Is it because I'm arthritic? Is it me? I'm sure a 5 year old would have no difficulty. I am physically unable to do this! These buttons are so tiny!" While all input modalities should be made more accessible, it is important that seniors can choose a secondary input method if the first does not suit them.

6. Avoid modifier keys. Like many PDA/phone hybrids, the KJAM does not feature a separate numeric keypad. Instead, numbers are entered on the QWERTY keyboard while holding down a modifier key. All participants made repeated errors while attempting to hold down this modifier key. Instead, separate keypads or virtual keypads devoted to a single set of characters should be used (e.g., one keypad for lower-case letters, one keypad for upper-case letters, one keypad for numbers).

7. Orient data structures towards personal, not business, use. Participants suggested adding diverse applications to the phone, as mentioned previously. These applications, at present, cater to business-class users. For example, the Windows Mobile Contact Manager prominently includes fields for title, department, secretary's phone number, fax numbers, and so on. These fields confused and distracted the seniors who simply wanted to add a friend and her phone number.

8. Avoid slide-out keyboards. Seniors had difficulty opening the slide-out keyboard. This especially included identifying the direction in which the case opened and how much pressure to apply. As an alternative, we suggest clamshell or integrated keyboards.

9. Carefully consider program and command naming. The terms seniors used to refer to programs and concepts varied. The terms reflected different mental models that did not match with how mobile phones currently work. For example, the team vigorously debated the name for the "Notes" program:

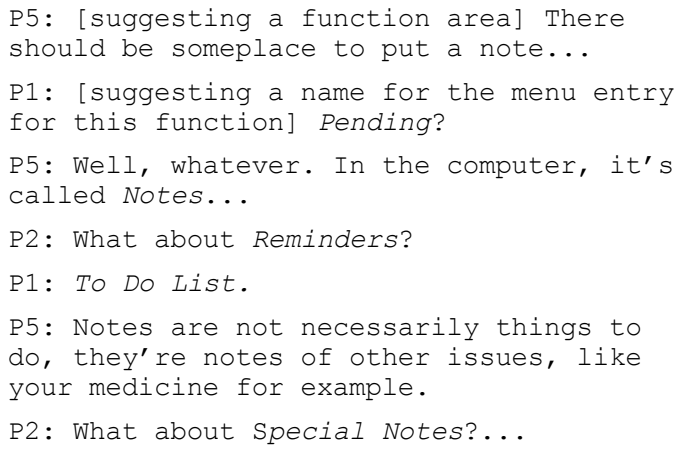

A similar discussion arose over the "How to Use This Phone" application; the seniors cited that "Help" did not indicate to them that clear directions would be provided about device 
operation. Designers should carefully consider the wording used for each program or command.

\section{DISCUSSION}

Participatory activities can help elicit valuable responses from participants that are difficult to obtain from large-scale studies such as surveys. With between 15 and 30 hours of face time with each senior, we could understand individual responses to the phone. For instance, P2 was quite positive about the phone throughout the entire process but revealed at the end that a family member recently died of too much radiation treatment. For this reason, P2 was scared to use the mobile phone because she perceived it to be emitting too much radiation.

\subsection{Design for Me, Us, or Them?}

One of the most important themes that occurred during the participatory activities is the conflict between three viewpoints on design. The first - "Design for Me" - is the notion that each individual, with their own set of abilities, should create a system that would be best for their own personal use. On the other hand, the "Design for Them" paradigm understands the need to design a system for a more general population (e.g., senior citizens) including people with a much greater diversity of abilities.

For example, in the exchange below, P5 demonstrates a "Design for Me" stance while P4 takes a "Design for Them" stance.

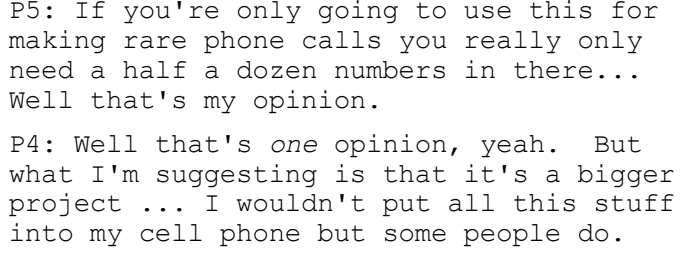

P5 argues that the Address Book should hold only a few numbers. $\mathrm{P} 4$, on the other hand, thinks that some other seniors will want to call many people using the mobile phone.

There is a middle ground between these two extremes - "Design for Us." P1, P2 and P3 shared this view. Their design decisions accounted for the abilities of the people directly on the design team with them, but did not generalize to seniors as a whole. For instance, P2 noted that while she does not play games, she thinks they should be included because P4 might like them.

Facilitators can choose to acknowledge and work with these differences in opinion, or promote a particular stance. Promoting a "Design for Me" stance may be required for some spectrum populations (e.g., children with autism) due to individualized deficits. Teams with explicit commercialization prospects, on the other hand, will want to adopt a "Design for Them" stance to promote product adoption. "Design for Us" is a more appropriate stance for individual programs or clinics that wish to create assistive technologies for organizational use, but not public consumption. The outcome of the process also depends greatly on the diversity of abilities of the team. If the team members are homogenous, it is likely that a "Design for Us" stance will be similar to the outcome of a "Design for Me" project. In our study, however, we noted that the diversity of the team and the "Design for Us" stance espoused by the team members yielded a generally applicable but less personalized system.

Facilitators should determine their goals and recruit accordingly. Choosing people with similar stances may create a productive team design environment due to shared understandings. However, selecting people with varying stances can promote more thoughtful and critical debate on particular design choices.

\subsection{Limitations of Participatory Activities}

There are some drawbacks to using participatory activities as a research tool. Our results may not generalize well. The considerations we offer are not guidelines because they have not been rigorously tested, although they were directly observed. In retrospect, our participatory methods could be improved as well.

We also found that seniors were better critics than designers. They could imagine using a phone and predict faults with it, but drawing interfaces was troublesome. At times, the team would often yield to P5's computer expertise. P5, in turn, would suggest designs that matched her understanding of her home computer.

Interestingly, the team did not show a clear preference for the software that they designed over the built-in software. This could be due to several reasons. First, both their software and the built-in software operate on the same device; it could be that the source of preference does not come from the user interface design as much as from the hardware design. Second, their skills and education about mobile phones could have increased their competence with all types of software, making the built-in software more accessible. Finally, there could have been a lack of creativity or execution during the paper prototyping or programming stages. In other words, it is possible that the system the team envisioned was not carried out faithfully. Our intuition, however, suggests that the team compromised on a design that they thought would match our expectations. They often referred to the process of participatory design as "learning the cell phone" and saw themselves not as creators of new software, but learners of old software. For this reason they created a design that, while simpler, was a traditional form-based application.

While this work focused on a mobile PDA/phone hybrid, the design guidelines can be judiciously applied to the class of mobile devices. For example, a wide range of devices, including tablet PCs and even walkie-talkies, include buttons on the sides of the hardware that seniors may accidentally press.

\section{CONCLUSION AND FUTURE WORK}

Mobile phones have the potential to positively impact several areas of seniors' lives, from supporting memory to promoting autonomous living. However, current phones seem to be targeting younger crowds and have designs that appeal to dexterous young adults. Previous focus groups and interviews with seniors about mobile phones have successfully engaged seniors in reflective processes. In this study, we instead chose to make seniors active codesigners of mobile phone software. We learned that software complexity can be handled; in fact, seniors desired multiple application domains and worked well with the camera and voice recorder functions. However, we found usability problems due to hardware and operating system design choices, thus creating faulty mental models. We also found that attitudes about mobile phones could be shaped by many sources, not simply usability concerns. For example, the problem may be due to a lack of critical-mass adoption, a lack of technical support, or fears of health risks from radiation.

This exploratory work suggests many questions that should be studied via other more rigorous methods. Laboratory testing of mobile device hardware designs for seniors, in particular, should be 
undertaken. We also need studies with larger samples of seniors to understand the prevalence of application-level concerns and desires. With more information about what makes mobile phones useful for seniors, we will be better able to design mobile systems that will meet their needs.

\section{ACKNOWLEDGMENTS}

Thanks to Kante Easley, Aha Blume, Dr. Kelly Murphy, Dr. David Ryan, Dr. Khai Truong, Dr. Alex Mihailidis, Dr. Jeremy Birnholtz, our paper mentor, and especially our participants.

\section{REFERENCES}

[1] Abeele, V. A. V. and Rompaey, V. V. Introducing humancentered research to game design: designing game concepts for and with senior citizens. Proc. CHI 2006 Extended Abstracts, ACM Press (2006), 1469-1474.

[2] Carroll, J. Scenario-based design: envisioning work and technology in system development. John Wiley \& Sons, Inc (1995). New York, NY, USA.

[3] Ellis, R. D. and Kurniawan, S. H. Increasing the usability of online information for older users: a case study in participatory design. International Journal of Human-Computer Interaction 12, 2 (2000), 263-276.

[4] Greenbaum, J. and M. Kyng Design at work: cooperative design of computer systems. Lawrence Erlbaum Associates, Inc. (2002). Mahwah, NJ, USA.

[5] Goodman, J., A. Dickinson, and Syme, A. Gathering requirements for mobile devices using focus groups with older people. Proceedings of the 2nd Cambridge Workshop on Universal Access and Assistive Technology (CWUAAT), Springer (2004).

[6] Kamssu, A. Global connectivity through wireless network technology: a possible solution for poor countries. International Journal of Mobile Communications 3, 3 (2005), 249-262.

[7] Keating, E., Nagai, E., Hadder, N., Kowalsky, J. The Role of the Mobile Phone in the Welfare of Aged and Disabled People. DoCoMo (2007), Retrieved from http://www.moba-

ken.jp/kennkyuu/chousa/2006/research06_05/finalreport_0605.pdf. Last accessed April 25, 2007.

[8] Kurniawan, S. and Zaphiris, P. Research-derived web design guidelines for older people. Proc. ASSETS 2005, ACM Press (2005), 129-135.

[9] Kurniawan, S. An exploratory study of how older women use mobile phones. Proc. UBICOMP 2006, Springer LNCS (2006), 103-122.

[10] Leonard, V. K., Jacko, J. A., and Pizzimenti, J. J. 2005. An exploratory investigation of handheld computer interaction for older adults with visual impairments. Proc. ASSETS 2005, ACM Press (2005), 12-19.
[11] Lewis, C. A model of mental model construction. Proc. CHI 1986, ACM Press (1986), 306-313.

[12] Liu, A. L., Hile, H., Kautz, H., Borriello, G., Brown, P. A., Harniss, M., and Johnson, K. Indoor wayfinding: developing a functional interface for individuals with cognitive impairments. Proc. ASSETS 2006, ACM Press (2006), 95-102.

[13] Moffatt, K., McGrenere, J., Purves, B., and Klawe, M. The participatory design of a sound and image enhanced daily planner for people with aphasia. Proc. CHI 2004, ACM Press (2004), 407-414.

[14] Muller, M. PICTIVE - an exploration in participatory design. Proc CHI 1991, ACM Press (1991), 225-231.

[15] Myers, B. A., Wobbrock, J. O., Yang, S., Yeung, B., Nichols, J., and Miller, R. Using handhelds to help people with motor impairments. Proc. ASSETS 2002, ACM Press (2002), 89-96.

[16] Nokia 6185. Retrieved from http://www.nokia.ca/english/products/6185/6185.asp. Last accessed May 23, 2007.

[17] Reuters. Mobile phone market seen to reach 2 billion users in '05. USA Today. Retrieved from http://www.usatoday.com/tech/wireless/phones/2005-01-19wless-outlook-05_x.htm. 2005. Last accessed May 23, 2007.

[18] Rosson, M. B., Carroll, J. M., Seals, C. D., and Lewis, T. L. Community design of community simulations. Proc. DIS 2002, ACM Press (2002), 75-83.

[19] Schulze, H. 2004. MEMOS: an interactive assistive system for prospective memory deficit compensation-architecture and functionality. Proc. ASSETS 2004, ACM Press (2004), 79-85.

[20] SilverPhone Easy5. Retrieved from http://www.silverphone.co.uk/mobilephone/info.php. 2004. Last accessed April 25, 2007.

[21] Sorrells, Z. Finding a mobile phone for grandma. Avec Mobile (2004). Retrieved from http://www.avecmobile.com/index.php?id=348. Last accessed April 25, 2007.

[22] Strothotte, T., Fritz, S., Michel, R., Raab, A., Petrie, H., Johnson, V., Reichert, L., and Schalt, A. Development of dialogue systems for a mobility aid for blind people: initial design and usability testing. Proc. ASSETS 1996, ACM Press (1996), 139-144.

[23] Tohidi, M., Buxton, W., Baecker, R., and Sellen, A. 2006. Getting the right design and the design right. Proc. CHI 2006, ACM Press (2006), 1243-1252.

[24] United Nations Division for Social Policy and Development. Report of the second world assembly on aging. Madrid, Spain: United Nations (2002).

[25] Wu, M., Baecker, R., and Richards, B. Participatory design of an orientation aid for amnesics. Proc. CHI 2005, ACM Press (2005), 511-520. 